J Ästhet Chir 2015 • 8:50-56

DOI 10.1007/s12631-014-0326-8

Online publiziert: 22. Mai 2015

(c) The Authors 2015. This article is published with open access at Springerlink.com

\title{
D. Rezek
}

Marien-Hospital gGmbH, Wesel, Deutschland

\section{Stellenwert der autologen Fetttransplantation in der Brustheilkunde}

\section{Fetttransfer: operative Prozedur in mehreren Schritten}

Die autologe Fetttransplantation ist eine operative Prozedur, bestehend aus:

- der Patientenselektion,

- der Fettgewinnung,

- der Aufbereitung des gewonnenen Gewebes,

- der Fetttransplantation sowie

- der Nachsorge der Patientin.

Der Erfolg dieser Operation ist von profunden Kenntnissen der Methode und einer sorgfältigen Ausführung jedes einzelnen Behandlungsschrittes abhängig. Gemessen wird er an Parametern wie Volumenstabilität, unbeeinträchtigter Beurteilbarkeit der Brust in bildgebenden Verfahren und onkologischer Sicherheit, vor allem, wenn autologes Fett in Regionen transplantiert wird, aus welchen vorher Brustkrebs entfernt worden ist.

Diskutieren wir den Stellenwert der autologen Fetttransplantation in der Brustheilkunde, müssen wir neben der Indikationsstellung und Patientenselektion jeden Einzelschritt der Methode und die Eigenschaften des Fettgewebes berücksichtigen.

\section{Eigenschaften des Fettgewebes} sätzlich weder formen, noch an jeden Defekt adaptieren.

Fett dagegen erscheint als das optimale Implantat. Denn Fett ist körpereigen, gut zugänglich, minimal-invasiv zu gewinnen, formbar, weich und einfach $\mathrm{zu}$ transplantieren. Darüber hinaus verfügt Fett neben füllenden auch über regenerative Eigenschaften.
Seit mehr als zehn Jahren wissen wir, dass es sich bei Fettgewebe nicht um einen inerten Füllstoff handelt, sondern um metabolisch aktives Gewebe, das aus einer heterogenen Zellpopulation besteht, den reifen Adipozyten, Fibroblasten, Blutgefäßen und adipösen mesenchymalen Stammzellen (ASC). Diese Fettstammzellen machen etwa $10 \%$ der Zellpopulation aus und haben die Fähigkeit, sich einerseits in verschiedene funktionelle Zellen zu differenzieren und andererseits Wachstumsfaktoren und Zytokine zu sezernieren [27, 36, $42,46]$.

\section{1) Es handelt sich bei Fettgewebe um metabolisch aktives Gewebe}

Adipozyten und ASC kommunizieren mit dem ortständigen Gewebe über Zytokine und stimulieren auf diese Weise die Angiogenese, reduzieren die Apoptose und modulieren die Immunantwort während der Reparationsvorgänge im Gewebe [24, 42].

Diese Eigenschaften sind für ein Transplantat sehr günstig, denn sie garantieren das Überleben des transplantierten Gewebes und den Volumenerhalt. Zugleich liegt gerade in diesen Eigenschaften das Risiko einer Tumorprogression oder Tumorentstehung.

\section{Onkologische Sicherheit}

In experimentellen Studien konnte nach Fetttransfer sowohl eine erhöhte Tumorzellvitalität, eine Zunahme der Proliferation und eine reduzierte Apoptose [14, 45] beobachtet werden, als auch das genaue Gegenteil, nämlich eine „down-regulation" der Zellsignale und eine Hemmung der Proliferation von Tumorgewebe [41].

In klinischen Studien konnte bisher weder eine Tumorneogenese, noch eine höhere Rate an lokoregionären Rezidiven durch Transplantation von autologem Fett in eine gesunde Brust beobachtet werden 


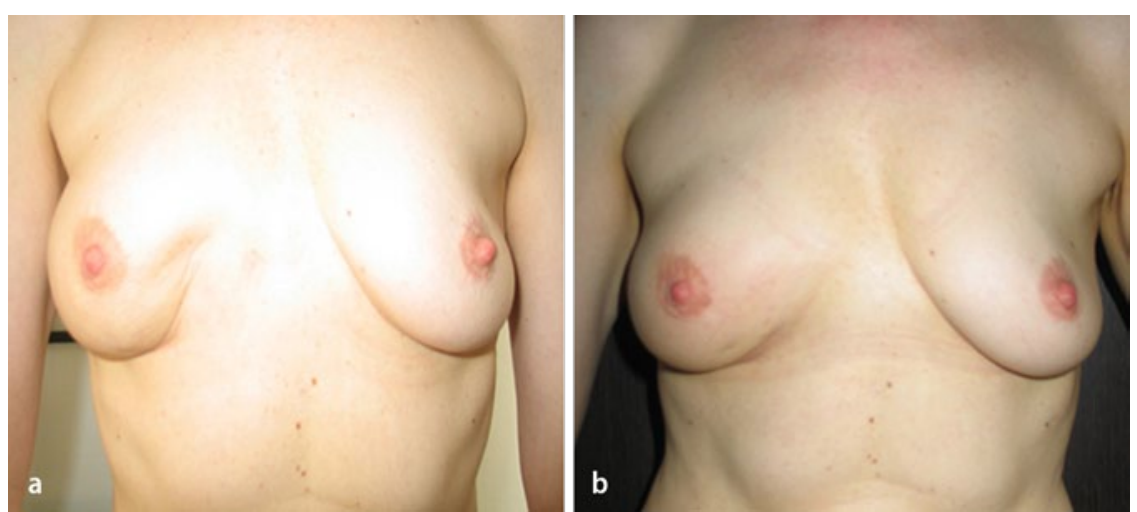

Abb. $1 \Delta$ a Patientin zwei Jahre nach brusterhaltender Therapie und Radiatio rechts unten innen. $\mathbf{b}$ Sechs Monate nach Fetttransfer rechts unten innen

$[5,7,16,29]$. Es gibt bisher eine einzige retrospektive Kohortenstudie, publiziert 2013 von Petit et al. [30], bei der eine Subgruppe mit einem besonderen Risikoprofil eine erhöhte Lokalrezidivrate nach Lipofilling zeigt. Es handelt sich um junge Patientinnen mit „high-grade“ duktalen (DIN) und lobulären (LIN) intraepithelialen Neoplasien, vor allem brusterhaltend operiert, einem hohen K-67-Proliferationsindex, unklarem Randstatus und kurzem Zeitintervall zwischen Krebsoperation und Li- pofilling. Auffällig ist, dass bei diesem Patientenkollektiv kein Lokalrezidiv auftrat, wenn der Lipotransfer länger als drei Jahre nach der Onkochirurgie durchgeführt wurde. Die Vermutung, dass möglicherweise residuale Tumorzellen durch das Lipofilling aktiviert wurden, bestätigt eine erst kürzlich online publizierte Studie [11]. Die Autoren haben das gleiche Studiendesign gewählt, das Zeitintervall zwischen der onkologischen Operation und dem Fetttransfer war aber mit 56 Monaten im Me- dian deutlich länger und die Ränder waren bei allen 211 eingeschlossenen Patientinnen sicher frei.

In dieser Studie konnte kein erhöhtes onkologisches Risiko durch autologe Fettransplantation bei Frauen nach Brustkrebsoperation nachgewiesen werden, auch nicht bei Patientinnen mit Vorstufen. Die Autoren analysierten und verglichen Literaturdaten von 1573 Frauen, die ein Lipofilling nach Operation eines Mammakarzinoms erhielten. Die Lokalrezidivrate war mit 2,9\% (0,95\% pro Jahr) nicht erhöht [11]. Diese Beobachtung wird ebenfalls durch die Publikation eines Rezidivs eines duktalen Mammakarzinoms bei einer jungen Patientin gestützt, die 24 Monate nach Mastektomie und Bestrahlung ein Lipofilling in die Brust erhielt. Vier Monate später sollte ein Expander eingesetzt werden. Die Mastektomienarbe wurde exzidiert und die histologische Aufarbeitung ergab ein Karzinomrezidiv mit Anordnung der Tumorzellen entlang der Transplantationstunnel [4]. Diese Patientin hatte zwei Jahre zuvor eine brusterhaltende Therapie (BET), wegen positiver Ränder (R1) eine

\section{Hier steht eine Anzeige.}


Mastektomie und Axilladissektion sowie Radiatio und Chemotherapie erhalten. Vor dem Transfer waren ein Rezidiv oder eine residuale Erkrankung nicht sorgfältig ausgeschlossen worden.

Es ist entscheidend, dass autologes Fett ausschließlich in sicher gesundes Gewebe transplantiert wird.

\section{Indikation}

Transfer von autologem Fett gewinnt in der Senologie immer mehr an Bedeutung. So zeigt eine Umfrage der britischen Chirurgen, dass 69\% der plastischen Chirurgen und $11 \%$ der Brustchirurgen, diese Operationsmethode regelmäßig nutzen und 60\% stimmen überein, dass der Nutzen dieser Methode das Risiko übertrifft [40].

\section{Korrektur von Kontur, Form und Volumendefekten nach BET}

Sowohl die Exzision großer benigner Tumoren, als auch die BET eines Mammakarzinoms kann zu Kontur-, Form oder Volumendefekten führen. Vor allem nach Segmentresektion, der derzeitigen Standardtherapie kleinerer Mammakarzinome [10], leiden die Patientinnen häufig unter den operations- und strahlungsbedingten Deformitäten [1, 17], insbesondere wenn keine adäquate onkoplastische Operation durchgeführt worden ist. $\bullet$ Abb. 1a zeigt eine 45-jährige Patientin zwei Jahre nach BET und $\mathbf{1 b}$ dieselbe Patientin sechs Monate nach Defektkorrektur durch Lipotransfer.

Aggraviert wird die Deformität häufig durch die auf die Operation folgende Bestrahlung, die zusätzlich die Hauttrophik beeinträchtigt. Hier bietet die autologe Fetttransplantation eine optimale Korrekturmöglichkeit. Vor allem die Arbeitsgruppe von Rigotti [35] zeigte beeindruckende Ergebnisse nach Lipofilling bei ausgeprägter Strahlendermatose.

\section{Korrektur des Weichteilmantels nach Mastektomie}

Bei der Mastektomie und Sofortrekonstruktion mit einem Expander, hauterhaltender Mastektomie („skin-/nipple-sparing mastectomy") und Implantateinlage sollte der Weichteilmantel aus Gründen der onkologischen Sicherheit dünn sein.

J Ästhet Chir 2015 • 8:50-56 DOI 10.1007/s12631-014-0326-8

(c) The Authors 2015. This article is published with open access at Springerlink.com

\section{Rezek \\ Stellenwert der autologen Fetttransplantation in der Brustheilkunde}

\section{Zusammenfassung}

Hintergrund. Die autologe Fetttransplantation ist eine operative Prozedur, bei der körpereigenes Fett übertragen wird, um die Form, das Volumen und die Oberflächenstruktur der Empfängerregion zu verbessern. Ziel und Methoden. Die vorliegende Arbeit beschreibt den Stellenwert und Stand der autologen Fetttransplantation. Dabei werden neben der Indikationsstellung und Patientenselektion jeder Einzelschritt der Methode und die Eigenschaften des Fettgewebes berücksichtigt.

Diskussion. Indiziert ist Lipofilling zur Korrektur von angeborenen Kontur-, Volumenund Formdefekten. Ebenso können erworbene Kontur- und Volumenprobleme nach Entfernung benigner Herde oder nach Reduktion und Augmentation mit Eigenfett korrigiert werden. Auch eine moderate Brustvergrößerung ist möglich. Bei Patientinnen nach Rekonstruktionen der Brust mit Implantaten oder Eigengewebe eignet sich autologes Fett zur Verstärkung des Weichteilmantels und Verbesserung der Kontur und der Hautperfusion insbesondere, wenn zuvor bestrahlt wor- den ist. Auch nach brusterhaltender Therapie kann nach einer Karenzzeit und sicherem Ausschluss residualer Erkrankung transplantiert werden. Das Fett sollte schonend gewonnen, gut aufbereitet und achtsam transplantiert werden. Postoperativ ist eine gute Nachsorge erforderlich.

Ergebnisse. Komplikationen nach Lipotransfer sind meist gering und treten aufgrund einer inadäquaten Selektion oder einer unsachgemäßen Durchführung auf. Fettgewebe darf ausschließlich in eine sicher gesunde Brust transplantiert werden. Der Erfolg dieser Operation ist von profunden Kenntnissen der Methode und einer sorgfältigen Ausführung jedes einzelnen Behandlungsschrittes abhängig. Gemessen wird er an Parametern wie Volumenstabilität, unbeeinträchtigter Beurteilbarkeit der Brust in bildgebenden Verfahren und onkologischer Sicherheit.

Schlüsselwörter

Lipofilling · Lipotransfer · Brustheilkunde . Mammakarzinom

\section{Importance of autologous fat transfer for breast augmentation}

\section{Abstract}

Background. Autologous fat transfer is a surgical procedure for transfer of a persons own body fat to improve the shape, the volume and the tissue surface of the recipient region. Aims and methods. This article describes the importance and state of the art of autologous fat transfer. Special emphasis is given to the indications and patient selection with a detailed description of each stage of the method and the characteristics of adipose tissue.

Discussion. Lipotransfer is indicated for correction of congenital defects in breast shape, volume and form. Acquired shape and volume problems after excision of benign lesions or after reduction mammaplasty and breast augmentation can also be corrected with autologous fat transfer. Even a modest augmentation of the breasts is possible. In patients after breast reconstruction with implants or flaps, autologous fat grafting can be used to ameliorate the covering soft tissue thickness and improve the contour and skin perfu- sion, especially after radiotherapy. Fat grafting can be performed even after breast conserving surgery after a latent period and when residual disease can definitely be excluded. Fat should be aspirated gently, well processed and carefully grafted. A meticulous follow-up monitoring is necessary postoperatively Results. Complications after lipotransfer are mostly slight and occur due to inadequate patient selection or inappropriate surgery. Fat may only be transplanted into a healthy breast. The success of this procedure is dependent on profound knowledge of the method and an accurate performance of every single step of the surgery, as measured by parameters, such as volume stability, unaffected assessment of the breast in radiological imaging and oncological safety

Keywords Lipofilling · Fat grafting · Senology · Breast cancer
Dies führt häufig zu Implantatkomplikationen. Auch hier eignet sich autologes Fett sehr gut zur Verstärkung des Weichteil- mantels und Verbesserung der Hautperfusion, insbesondere wenn bestrahlt worden ist $[35,38]$. In $\bullet$ Abb. 2 wird eine Patien- 

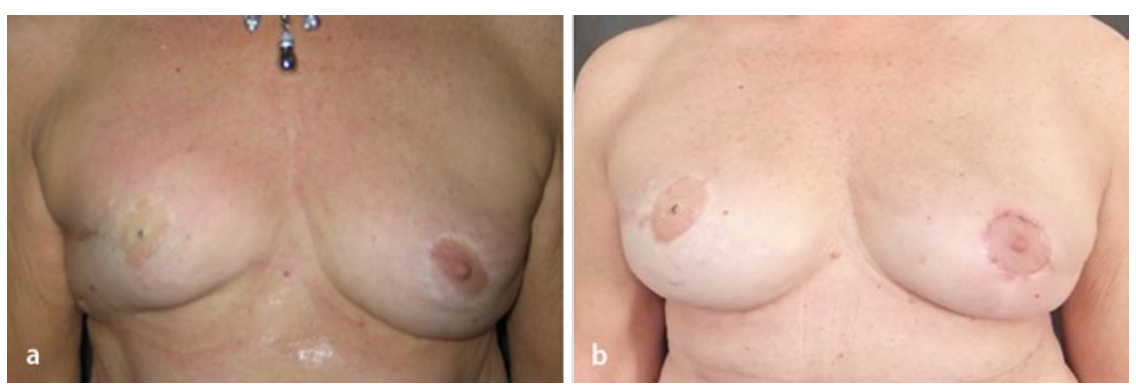

Abb. 2 - Implantat nach Mastektomie der rechten Mamma. a Patientin acht Jahre nach Ablatio und Implantat rechts. b Patientin zwölf Monate nach Fetttransfer rechts, Mastopexie links

tin gezeigt, die nach Mastektomie der rechten Mamma einen sehr dünnen Weichteilmantel über dem Implantat und eine deutliche Anisomastie hat. Ein Jahr nach Lipotransfer ist die Anisomastie weitgehend behoben, die Patientin hat zusätzlich noch eine Narbenkorrektur der kontralateralen Mastopexienarbe durchführen lassen.

Auch nach Mastektomie und Rekonstruktion mit gestielten oder freien Lappen kommt es zu Form- und Konturunregelmäßigkeiten, diese können gut mit Eigenfett korrigiert werden [18, 39].

\section{Korrektur kongenitaler und erworbener Brustanomalien und bei Volumenmangel}

Lipofilling ist zur Korrektur kongenitaler und erworbener Brust- und Thoraxwandanomalien wie Poland-Syndrom, Pectus excavatum, tuberöser Brust und Anisomastie geeignet $[7,16]$.

Kontur- und Volumenprobleme des oberen Brustpols nach Reduktion und/ oder Mastopexie, Konturprobleme nach
Augmentation, wie „rippling“ oder Kapselkontraktur können sehr gut mit Eigenfett korrigiert werden [7, 21]. • Abb. 3 zeigt die Korrektur von „rippling“ nach einer epipektoralen Augmentation.

Auch eine moderate Brustaugmentation ohne sichtbare Narben kann mit Eigenfett alleine erreicht werden [2, 5]. Die 36-jährige Patientin in $\bullet$ Abb. 4 wünschte eine moderate Augmentation ohne sichtbare Narben.

\section{Patientenselektion und -aufklärung}

Vor einem Lipotransfer sollten Komorbiditäten, Lebensgewohnheiten und die Compliance der Patientin berücksichtigt werden. Die Patienten sollten nach dem Transfer nicht rauchen und mindestens sechs Monate ihr Gewicht stabil halten.

Aufzuklären ist darüber, dass es möglicherweise potenzielle Risiken dieser Methode gibt, die zum jetzigen Zeitpunkt noch unbekannt sind, sowie darüber, dass der Erfolg nicht garantiert werden kann und der Eingriff eventuell wiederholt werden muss, um das gewünschte Ergebnis zu erzielen.

\section{I) Nach brusterhaltender Therapie sollte eine Karenzzeit von zwei bis drei Jahren eingehalten werden}

Bei Patientinnen nach BET eines Mammakarzinoms oder einer Vorstufe sollten der Nutzen und das individuelle Risiko aufgrund der Tumorbiologie diskutiert und eine Karenzzeit von mindestens zwei, besser drei Jahren eingehalten werden $[9,11,15,34]$.

\section{Kontraindikation}

Eine autologe Fetttransplantation sollte bei akuten Erkrankungen der Empfänger- oder Spenderregion, bei Problemen der Blutgerinnung und in der Schwangerschaft nicht erfolgen.

\section{Prozedur der Fettgewinnung}

Lipotransfer ist eine operative Prozedur, die aus der Entnahme von Fettgewebe, der Aufbereitung des gewonnenen Fetts und der Transplantation besteht. Daher sollte die Durchführung unter sterilen Bedingungen erfolgen. Geschlossene Systeme sind zu bevorzugen, denn Luftexposition führt neben einer möglichen Kontamination zu Stress und daraus resultierender Vitalitätsreduktion des Trans-

\section{Hier steht eine Anzeige.}



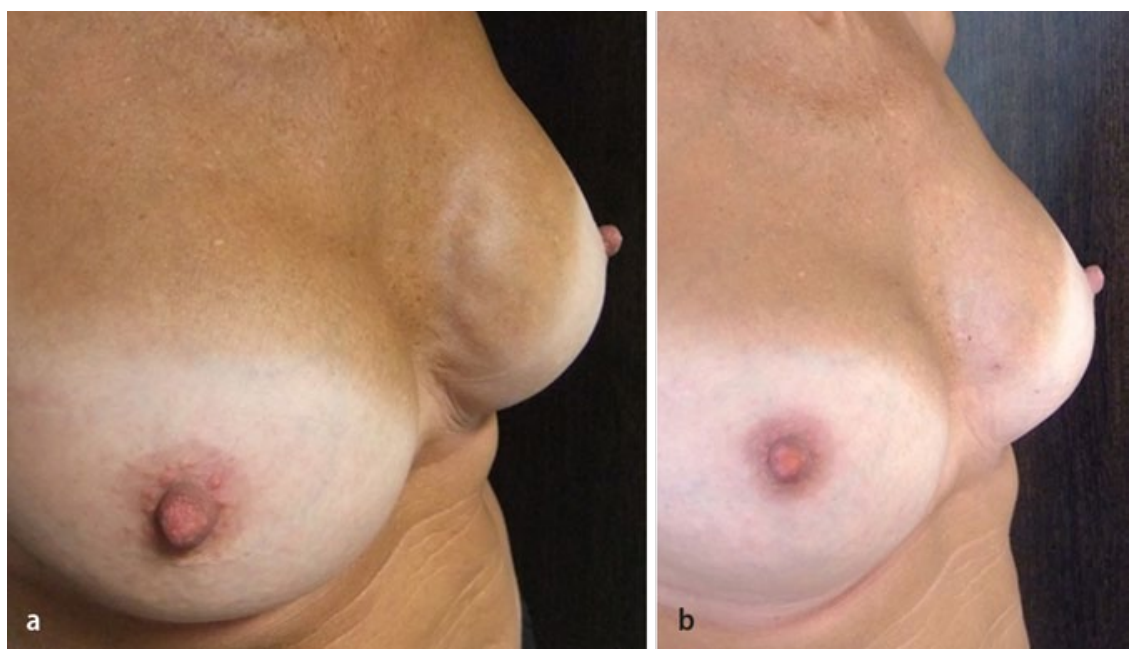

Abb. $3 \Delta$ Korrektur von "rippling“ nach epipektoraler Augmentation. a Patientin nach epipektoraler Augmentation und „,rippling". b Nach Korrektur mit autologem Fett
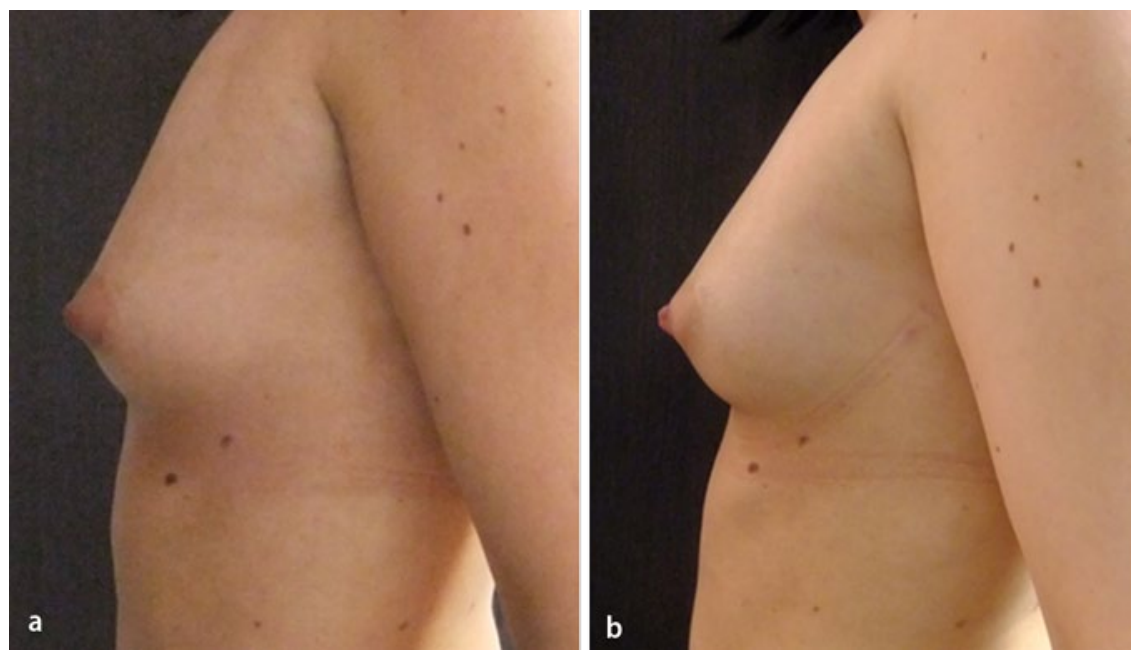

Abb. 4 \ Patientin mit Wunsch nach natürlicher, narbenfreier Augmentation. a Präoperativ. b Nach Augmentation mit autologem Fett

plantats [19]. Ob die Spenderstelle für die gewonnene Zellqualität eine Rolle spielt, wird kontrovers diskutiert [13, 37]. Daher kann die Spenderstelle nach Absprache mit der Patientin entsprechend deren Wunsch oder Fettdepot gewählt werden.

Die in der Tumeszenzlösung verwendeten Lokalanästhetika verringern in $\mathrm{Ab}$ hängigkeit von der Expositionsdauer die Vitalität der aspirierten Zellen, vor allem der Präadipozyten. Dabei hat Lidocain den geringsten zytotoxischen Einfluss [12]. Deshalb ist eine Lösung aus Kochsalz und Adrenalin zu bevorzugen, da Adrenalin keinen Einfluss auf die Zellvitalität hat.

\section{Saugtechnik}

Die Aspiration erfolgt optimalerweise mit kontrolliertem Sog und reduziertem Vaku- um zwischen $-0,5$ bis $-0,55$ bar. Ist der Sog zu hoch, können die Zellmembranen rupturieren [22]. Mikrokanülen mit mehreren Öffnungen sind zu favorisieren, da sie die Kollektion kleiner Lobuli erleichtern, die besser revaskularisiert werden [25].

\section{Aufbereitung des Fetts}

Die Aufbereitung des Aspirats dient der Reinigung, der Entfernung von Zelldetritus, Blut, Flüssigkeit und Öl, um ein homogenes Transplantat zu gewinnen. Denn ein Transfer von Öl führt zu Nekrosen, und eine Transplantation von Blut und Detritus führt zur Entzündung und Hemmung der Angiogenese [6]. Ob eine schonende Zentrifugierung und die Filtration gleichwertig sind, oder eine Aufbereitungsmethode der anderen überle- gen ist, wird derzeit untersucht [20]. Es gibt zahlreiche Prozessierungsmethoden, die dazu dienen sollen, die Volumenpersistenz zu verbessern. Hierzu werden z. B. „Platelet-rich-plasma“(PRP)-Insulin, oder isolierte und enzymatisch aufbereitete Stammzellen (ASC) dem Lipoaspirat beigefügt. Die Beimengung von aufbereiteten ASC wird zellassistierter Lipotransfer (CAL) genannt [44]. In prospektiven klinischen Studien konnte jedoch bisher kein Benefit dieser Methoden nachgewiesen werden [28].

\section{Fetttransfer}

Die Transplantation in die Brust sollte schonend mit stumpfen dünnen Kanülen $(1,2-3 \mathrm{~mm})$ in mehreren Schichten, retrograd unter Freigabe von kleinen Fettmengen erfolgen. Auch sollten nicht $\mathrm{zu}$ viele Tunnel geschaffen werden, denn übermäßiger mechanischer Stress führt zur Entzündung und zu Ödemen und damit zur verstärkten Resorption des Transplantats. Die transferierte Menge sollte der Gewebeelastizität angepasst werden, denn eine Überfüllung der Empfängerregion kann zu einer venösen Obstruktion führen. Je nach Gewebeelastizität können $50-400 \mathrm{~cm}^{3}$ in einer Sitzung sicher transferiert werden [19].Die Transplantation von Fett kann subkutan, subglandulär und intramuskulär erfolgen.

) Die Transplantation von Fett kann subkutan, subglandulär und intramuskulär erfolgen

Eine intraglanduläre Applikation sollte vermieden werden,

- weil dies zu einer erschwerten Beurteilung des Drüsengewebes in der Bildgebung führen kann,

- weil es zu einer postoperativen Infektion kommen kann, da die Milchgänge mit Mikroorganismen besiedelt sind, und

- wegen des Risikos einer malignen Proliferation von Tumorzellen durch Injektion von Stammzellen in ein potenzielles Tumorbett [26]. 

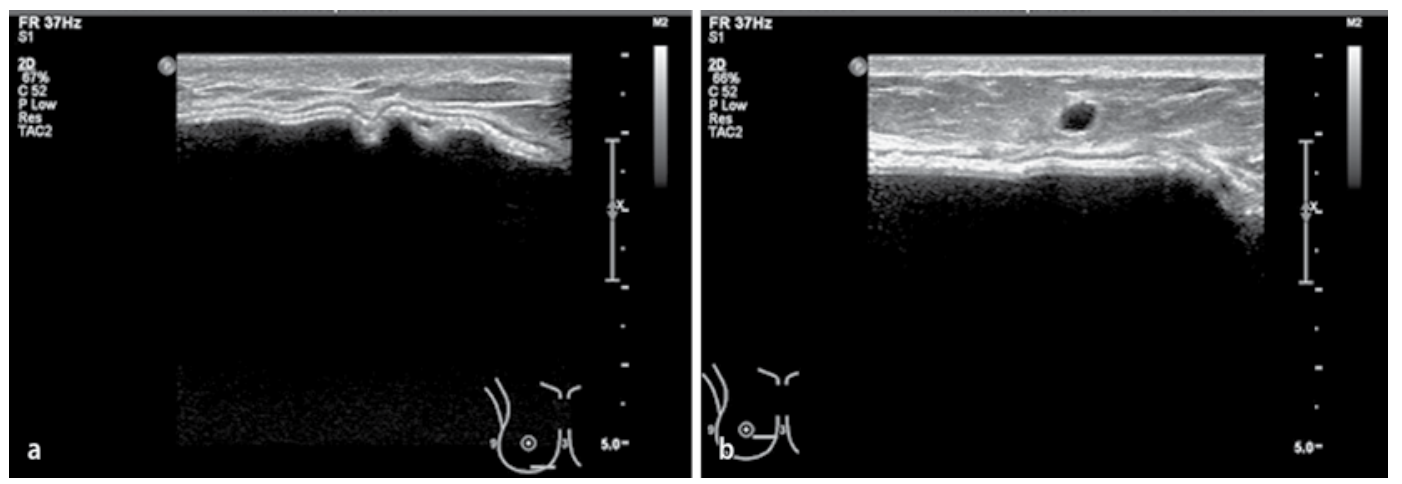

Abb. $5<$ Diagnostik von Herden in der Brust nach Fettransfer. a Dünner Hautmantel über dem Implantat nach Mastektomie rechts. $\mathbf{b}$ Ultraschallbild bei gleicher Patientin 15 Monate nach Fetttransfer über das Implantat, mit kleiner Ölzyste

\section{Postoperatives Management}

Frisch transplantiertes Fett sollte nicht komprimiert werden, die Spenderregion ist jedoch mit einem Kompressionsmieder zu bekleiden [33].

Wie bei jedem operativen Eingriff an der Brust ist eine perioperative Antibiose ratsam. Eine frühe Wundinspektion sollte erfolgen, um Komplikationen wie Infektionen, Hämatome und drohende Nekrosen früh zu erkennen. Danach können im Konsens mit der Patientin regelmäßige Kontrollen vereinbart werden, um die Volumenpersistenz zu beurteilen und gegebenenfalls weitere Transfers zu planen.

Die Sonographie ist eine gute Methode zur Diagnostik von Herden in der Brust. Vor einer Fettransplantation können im Ultraschall die Brustdrüse und das Fettgewebe oder der Weichteilmantel über dem Implantat gut beurteilt werden. Nach dem Eingriff können sowohl das transplantierte Volumen, die Veränderung des Weichteilmantels, als auch Ölzysten oder Fettnekrosen dargestellt und beurteilt werden ( $\bullet$ Abb. 5).

\section{Patientensicherheit}

Vor jeder autologen Fetttransplantation sollte eine Fotodokumentation der Brust und der Spenderstelle erfolgen. Eine Mammographie wird bei Patientinnen über 40 Jahren vor der Durchführung eines Fetttransfers empfohlen [34]. Bei jüngeren Patientinnen ohne familiäre Mammakarzinombelastung ist der Ultraschall der Brust und der axillären Lymphabflußwege ausreichend. Bei Patientinnen, die an einem Mammakarzinom erkrankt waren, ist zu berücksichtigen, dass die Darstellung eines Lokalrezidivs in der Mammographie in den ersten sechs Monaten nach dem Transfer erschwert sein könnte, ebenso wie nach anderen Operationsverfahren auch. Eine Mammographie ist daher frühestens sechs Monate nach dem Transfer sinnvoll $[3,31,43]$.

\section{Komplikationen}

Die autologe Fetttransplantation ist eine komplikationsarme Operationsmethode. Schwerwiegende Komplikationen wie revisionsbedürftige Nekrosen, Abszessbildung oder Pneumothorax sind hauptsächlich aufgrund der Injektion großer Volumina und unsachgemäßer Durchführung der Operation durch ungeübte Chirurgen entstanden und in Reviews beschrieben [23].

\section{Veränderungen in der radiologischen Bildgebung}

Nach autologer Fetttransplantation treten nicht mehr Veränderungen im Sinn von Kalzifikationen, Ölzysten oder Fettnekrosen auf als nach anderen Eingriffen an der Brust, wie Reduktions- oder Lappenplastiken $[3,5,43]$.

Sonographisch lassen sich vor allem in den ersten sechs Monaten Ölzysten darstellen, Kalzifikationen und Fettnekrosen sind in der Mammographie nach etwa einem Jahr darstellbar und stabilisieren sich nach zwei Jahren [32].

Mit den heutigen radiologischen Untersuchungsmethoden sind diese Veränderungen gut von Tumoren oder Lokalrezidiven zu unterscheiden.

\section{Fazit für die Praxis}

- Die autologe Fetttransplantation ist zur Korrektur von Form, Kontur und Volumen angeborener und erworbener Defekte der weiblichen Brust so- wie zur moderaten Augmentation indiziert.

- Bei Patientinnen nach Mastektomie und Implantatrekonstruktion eignet sich die Fetttransplantation zur Verstärkung des Weichteilmantels und Verbesserung der Hautdurchblutung, bei Patientinnen nach Mastektomie und Eigengewebsrekonstruktion zur Konturverbesserung.

- Auch bei Patientinnen nach brusterhaltender Therapie eines invasiven Mammakarzinoms oder von Vorstufen kann Eigenfett eingesetzt werden, wenn eine residuale Erkrankung sicher ausgeschlossen ist.

- Bei Mammakarzinompatientinnen sollte eine Karenzzeit von mindestens 24 Monaten, je nach Tumorbiologie besser 36 Monaten, eingehalten werden.

- Je länger das Zeitintervall zwischen der Onkochirurgie und dem Fettransfer, desto höher ist die onkologische Sicherheit.

- Jeder einzelne Operationsschritt, die Patientenselektion, Fettgewinnung, Aufbereitung, der Transfer und die Nachsorge, sollte sorgfältig und nur von geübten Operateuren durchgeführt werden.

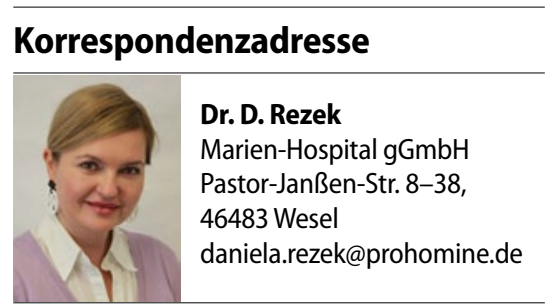

Einhaltung ethischer Richtlinien

Interessenkonflikt. D. Rezek gibt an, dass kein Interessenkonflikt besteht. 
Alle Patienten, die über Bildmaterial oder anderweitige Angaben innerhalb des Manuskripts zu identifizieren sind, haben hierzu ihre schriftliche Einwilligung gegeben. Im Falle von nicht mündigen Patienten liegt die Einwilligung eines Erziehungsberechtigten oder des gesetzlich bestellten Betreuers vor. Dieser Beitrag beinhaltet keine Studien an Menschen oder Tieren.

\section{Open Access}

This article is distributed under the terms of the Creative Commons Attribution 4.0 International License (http://creativecommons.org/licenses/by/4.0/), which permits unrestricted use, distribution, and reproduction in any medium, provided you give appropriate credit to the original author(s) and the source, provide a link to the Creative Commons license, and indicate if changes were made.

\section{Literatur}

1. Asgeirsson KS, Rasheed T, Mc Culley SJ, Macmillan RD (2005) Oncological and cosmetic outcomes of oncoplastic breast conserving surgery. Eur J Surg Oncol 31:817-823

2. Bircoll M (1987) Cosmetic breast augmentation utilising autologous fat and liposuktion techniques. Plast Reconstr Surg 79(2):267-271

3. Chala LF et al (2004) Fat necrosis of the breast: mammographic, sonographic, computed tomography, and magnetic resonance imaging findings. Curr Probl Diagn Radiol 33:106-126

4. Chaput B, Foucras L, Gullec S et al (2013) Recurrence of an invasive ductal breast carcinoma 4 month after autologous fat grafting. Plast Reconstr Surg 131:123e-124e

5. Coleman SR, Saboeiro AP (2007) Fat grafting to the breast revisited: safety and efficacy. Plast Reconstr Surg 119:775-785

6. Conde-Green A, Baptista LS, Amorin NF de et al (2010) Effects of centrifugation on cell composition and viability of aspirated adipose

tissue processed for transplantations. Aesthet Surg J 30:249-255

7. Delay E, Garson S, Tousson G, Sinna R (2009) Fat injection to the breast: technique, results, and indications based on 880 procedures over 10 years. Aesthet Surg J 29(5):360-376

8. Delay $\mathrm{E}$ (2006) Lipomodelling oft the reconstructed breast. In: Spear SL (Hrsg) Surgery of the breast - principles and art, 2. Aufl. Lippincott Williams\&Wilkins, Philadelphia, S 930-946

9. Dieterich M, Dieterich H, Gerber B (2012) Lipofilling und Hyaluronsäure in der ästhetischen und rekonstruktiven Mammachirurgie. Hintergründe, Indikationen und onkologische Aspekte. Gynäkol Prax 36:715-729

10. Early Breast Cancer Trialists' Collaborative Group (EBCTCG), Darby S, McGale P et al (2011) Effect of radiotherapy after breast-conserving surgery on 10-year recurrence and 15-year breast cancer death; meta-analysis of individual patient data for 10,801 women in 17 randomised trials. Lancet 9804:1707-1716

11. Gale KL, Rakha EA, Ball G, Tan VKM et al (2015) A case controlled study of the oncological safety of fat grafting. Plast Reconstr Surg (online first)

12. Girard AC, Festy F, Roche R (2013) Local anesthetics: use and effects in autologous fat grafting. Surg Curr Res 3:142
13. Heimburg D von, Hemmrich $K$, Haydarioglu S et al (2004) Comparison of viable cell yield from excised versus aspirated adipose tissue. Cells Tissues Organs 178:87-92

14. Hsu VM, Stransky CA, Bucky LP, Percec I (2012) Fat grafting's past, present, and future: why adipose tissue is emerging as a critical link to the advancement of regenerative medicine. Aesthet Surg J 32(7):892-899

15. Ihrai T, Georgiou C, Machiavello JC et al (2013) Autologous fat grafting and breast cancer recurrences: retrospective analysis of a series of 100 procedures in 64patients. J Plast Surg Hand Surg 47:273-275

16. Illouz YG, Serodimas A (2009) Autologous fat transplantations to the breast; a personal technique with 25 years of experience. Aestetic Plast Surg 33(05):706-715

17. Kelly DA, Wood BC, Knoll GM, Chang SC et al (2012) Outcome analysis of 541 undergoing breast conservation therapy. Ann Plast Surg 68:435-437

18. Kanchwala SK, Glatt BS, Conant EF, Bucky LP (2009) Autologous fat grafting to the reconstructed breast: the management of acquired contour deformities. Plast Reconst Surg 124:409-418

19. Largo RD, Tchang LA, Mele V et al (2013) Efficacy, safety and complications of autologous fat grafting to healthy breast tissue: asystematic review. $J$ Plast Reconstr Aesth Surg 67(4):437-448

20. Mestak O, Sukop A, Hsueh YS et al (2014) Centrifugation versus PureGraft for fatgrafting to the breast after breast conserving therapy. World J Surg Oncol 12:178

21. Missana MC, Laurent I, Barreau L et al (2007) Autologous fat transfer in reconsctructive breast surgery: indications, technique and results. Eur J Surg Oncol 33:685-690

22. Mojallal A, Auxenfans C, Lequeux C et al (2008) Influence of negative pressure when harvesting adipose tissue on cell yield of the stromal-vascular fraction. Biomed Mater Eng 18:193-197

23. Mu DL, Luan J, Mu L, Xin MQ (2009) Breast augmentation by autologous fat injection grafting: management and clinical analysis of complications. Ann Plast Surg 63:124-127

24. Montrescu ER, Rio MC (2008) Cancer cells, adipocytes and matrix metalloproteinase 11: a vicious tumor progression cycle. Biolog Chem 389(8):1037-1041

25. Nguyen A, Pasyk KA, Bouvier TN et al (1990) Comparative study of survival of autologus adipose tissue taken and transplanted by different techniques. Plast Reconstr Surg 85:378-386

26. Pallua N, Kim B-S (2013) Brustrekonstruktion mittels autologer Fetttransplantation - Ein sicheres Verfahren? J Aesthet Chir 6:160-165

27. Pearl RA, Leedham SJ, Pacifico MD (2012) The safety of autologous fat transfer in breast cancer: lessons from stem cell biology. J Plast Recontr Aesth Surg 65(3):283-288

28. Peltoniemi HH, Salmi A, Miettinen S et al (2013) Stem cell enrichment does not warrant a higher graft survival in lipofilling of the the breast: a prospective comparative study. 66:1494-1503

29. Petit JY, Lohsiriwat V, Clough KB et al (2011) The oncologic outcome and immediate surgical complications of lipofilling in breast cancer patients: a multicenter study-milan-paris-lyon experience of 646 lipofilling procedures. Plast Reconstr Surg 128:341-346

30. Petit JY, Rietjens M, Botteri E, Rotmensz $\mathrm{N}$ et al (2013) Evaluation fo fat graftig safety in patients with intra epithelial neoplasia: a matched-cohort study. Ann Oncol 24(6):1479-1484
31. Pierrefeu-Lagrange AC, Delay $E$, Guerin $N$ et al (2006) Radiological evaluation of breasts reconstructed with lipomodeling. Ann Chir Plast Esthet 51:18-28

32. Pulagam SR, Pulton T, Mamounas EP (2006) Long term clinical and radiologic results with autologous fat transplantation for breast augmentation: case reports and review of the literature. Breast 12(1):63-65

33. Rennekampff HO, Reimers K, Gabka CJ et al (2010) [Current perspective and limitations of autologous fat transplantation - „, consensus meeting" of the German Society of Plastic, Reconstructive and Aesthetic Surgeons at Hannover; September 2009]. Handchir Microchir Plast Chir 42802:137-142

34. Rezek D, Dieterich M; Arbeitsgemeinschaft für ästhetische, plastische und wiederherstellende Operationsverfahren in der Gynäkologie e. V. in der Deutschen Gesellschaft für Gynäkologie und Geburtshilfe (DGGG) (2013) Empfehlungen der AWOgyn zum Lipofilling. Lipofilling im Rahmen der ästhetischen und rekonstruktiven Brustchirurgie. Version 2013/2014

35. Rigotti G, Marchi A, Galie M et al (2007) Clinical treatment of radiotherapy tissue damage by lipoaspirate transplant: a healing process mediated by adipose-derived adult stem cells. Plast Reconstr Surg 119:1409-1422 (discussion 1423-1424)

36. Rigotti G, Marchi A, Sbarbati A (2009) Adipose-derived mesenchymal stem cells: past, present, and future. Aesth Plast Surg 33(3):271-273

37. Rohrich RJ, Sorokin ES, Brown SA (2004) Search of improved fat transfer viability: a quantitative analysis of the role of centrifugation and harvesting. Plast Reconstr Surg 113:391e-397e

38. Serra-Renom JM, Munoz-Olmo JL, Serra-Mestre JM (2010) Fat grafting in postmastektomybreast reconstruction with expanders and prostheses in patients who have received radiotherapy: formation of new subcutaneous tissue. Plast Reconstr Surg 125(1):12-18

39. Sinna R, Delay E et al (2010) Breast fat grafting (Lipomodelling) after extended latissimus dorsi flat breast reconstruction: a pray lineary report of 200 consecutive cases. J Plast Reconstr Aesthet Surg 63:1769-1777

40. Skillmann J, Hardwicke J, Whisker L, England D (2013) Attitudes of UK breast and plastic surgeons to lipomodelling in breast surgery. Breast 22(6):1200-1204

41. Sun B, Roh KH, Park JR et al (2009) Therapeutic potential of mesenchymal stromal cells in a mouse breast cancer metastasis model. Cytotherapy 11:289-298

42. Vona-Davis L, Rose DP (2007) Adipokines as endocrine, paracrine, and autocrine factors in breast cancer risk and progression. Endocr Relat Cancer 14(2):189-206

43. Veber M, Tourasse C, Delay E (2011) Radiographic findings after breast augmentation by autologous fat transfer. Plast Reconstr Surg 127:1289-1299

44. Yoshimura K, Sato K, Aoi N et al (2008) Cell-assisted lipotransfer for cosmetic breast augmentation: supportive use of adipose-derived stem/stromal cells. Aesthetic Plast Surg 32:48-55 (discussion 56-47)

45. Zimmerlin L, Donnenberg AD, Rubin JP et al (2011) Regenerative therapy and cancer: in vitro and in vivo studies of the interaction between adiposederived stem cells and breast cancer cells from clinical isolates. Tissue Eng Part A 17:93-106

46. Zuk PA, Zhu M, Ashjian P et al (2002) Human adipose tissue is a source of multipotent stem cells. Mol Biol Cell 13:4279-4295 